\title{
Foam posturography - Standing on foam is not equivalent to standing with decreased rapidly adapting mechanoreceptive sensation
}

\author{
Patel $\mathrm{M}^{1}$, Fransson $\mathrm{PA}^{1}$, Johansson $\mathrm{R}^{1,2}$, Magnusson $\mathrm{M}^{1}$. \\ ${ }^{1}$ Department of Clinical Sciences, Lund, Lund University, S-221 85 Lund, Sweden. \\ ${ }^{2}$ Department of Automatic Control, Lund University, S-221 00 Lund, Sweden
}

Address for correspondence:

Fransson PA, Department of Clinical Sciences, Lund,

Division Otorhinolaryngology Head and Neck surgery,

Lund University,

S-221 85 Lund, Sweden

Phone: Int. + 46, 46 171770; Fax: Int. + 46, 462110968

E-mail: Per-Anders.Fransson@med.lu.se 


\section{Abstract}

Standing on a foam surface is believed to exaggerate balance deficits by decreasing the reliability of somatosensory information from cutaneous mechanoreceptors on the plantar soles (i.e. base of feet) and by altering the effectiveness of ankle torque. The aim was to further document the nature of foam posturography testing by comparing between standing on foam and standing with decreased Rapidly Adapting Mechanoreceptive Sensation (RAMS).

Sixteen healthy adults (mean age 20.8 years) were tested with posturography, standing with eyes open and closed on a solid surface and on foam, with and without decreased plantar RAMS. Standing balance was measured as torque variance and further analyzed by being divided into three spectral categories. Plantar cutaneous hypothermic anesthesia by ice-cooling was used to decrease RAMS. Plantar mechanoreceptive sensation was precisely determined with tactile sensitivity and vibration perception tests

Vibration perception was significantly decreased by hypothermic anesthesia, but tactile sensitivity was not. The anterior-posterior torque variance was significantly larger for frequencies less than $0.1 \mathrm{~Hz}$ under eyes closed conditions when standing on a solid surface with decreased RAMS compared to normal sensation. No effect of decreased RAMS was seen with eyes open on a solid surface, nor on foam with eyes open or closed. Decreased RAMS produced body sway responses on a solid surface that were different in spectral composition, amplitude, direction and that responded differently to vision compared with standing on foam.

Hence, this study showed that RAMS contributes to postural control but reduction of RAMS does not produce a similar challenge as standing on foam.

Key Words: Postural stability; Plantar sensation; Foam; Clinical test; Cooling 


\section{Introduction}

Standing postural control depends upon continual integration of sensory inputs from visual, vestibular and somatosensory receptors (proprioceptors and mechanoreceptors) by the Central Nervous System (CNS), to assess position and motion of the body (Johansson and Magnusson 1991; Massion 1994). However, unavailable, defective or inappropriate information from any one of the sensory receptors can result in instability due to mismatch between incoming sensory signals (Bloem et al. 2000; Bloem et al. 2002). The CNS is able to compensate to some extent by relying more upon information from the remaining, correctly functioning receptors, termed sensory re-weighting (Allum and Honegger 1998). An important source of somatosensory information comes from plantar (i.e. soles of the feet) mechanoreceptors (Pyykko et al. 1990), and this is particularly important when balance is perturbed (Stal et al. 2003). These mechanoreceptors are either slowly or rapidly adapting. The slowly adapting plantar mechanoreceptors provide information about how the pressures are spatially and sequentially distributed on the skin (Kavounoudias et al. 1998), i.e. the foot sole - surface interaction. The rapidly adapting mechanoreceptors provide information about pressure amplitude and changes of pressure exerted on the skin (Kavounoudias et al. 1998), i.e., the fast changes of posture which are used for postural control (Kennedy and Inglis 2002).

Assessing unperturbed standing on a firm surface often lacks the specificity for distinguishing healthy patients from those with balance disorders (Johansson and Magnusson 1991), and therefore a number of balance perturbing methods have been devised to increase demand on the postural control mechanisms. One common method has been to have patients stand on a compliant surface such as foam, particularly in the case of suspected vestibular disorders (Furman 1995; Fujimoto et al. 2009). When standing on a foam surface, the relative contributions of the visual, vestibular and somatosensory inputs change (Enbom et al. 1991; Furman 1995). Along with reducing the effectiveness of ankle torque for postural stabilization due to its visco-elasticity (Horak and Hlavacka 2001; MacLellan and Patla 2006; Patel et al. 
2008a), foam surfaces are believed to challenge postural control by decreasing the reliability of sensory information from plantar mechanoreceptors (Wu and Chiang 1997; Perry et al. 2000). However, the degree to which mechanoreceptive information is affected by foam surfaces is unknown. To fully grasp the implications of poor stability while standing on foam and its usefulness in the assessment of vestibular disorders, a comprehensive evaluation of how foam challenges standing postural control is necessary.

Previous investigations (Patel et al. 2008a; Patel et al. 2008b) have showed that the mechanical properties of the foam surface, e.g., the foam surface density and the extent to which the foam material compresses under a given force, are significantly related to the resulting stability challenge. The present study aimed to extend upon previous work by investigating the contribution of decreased Rapidly Adapting Mechanoreceptive Sensation (RAMS) to the balance challenge when standing on foam. The study objective was to compare the postural responses when standing on a solid surface with a foam surface, both when RAMS feedback was fully available and reliable and when decreased by hypothermic ischemia (Perry et al. 2000; Stal et al. 2003). Hypothermic ischemia involves cooling the plantar sole with ice or ice water, causing a gain reduction in the mechano-electrical transfer functions rather than an impairment of pulse transmission in terminal nerve branches (Kunesch et al. 1987). Hence, hypothermic ischemia restricts information from plantar mechanoreceptors, and this sensation decrease can be objectively assessed using monofilaments and biothesiometer. Considering the dominant role played by visual information when standing on foam (Fransson et al. 2007), the experiments were performed both with eyes open and eyes closed.

\section{Materials and Methods}

\subsection{Participants}

All 16 participants (12 male and 4 female) were naïve healthy adults between the ages of 18 and 26 years (mean 20.8 years with a standard deviation (SD) of 2.0 years). Their average 
mass was $83.0 \mathrm{~kg}$ (SD $15.0 \mathrm{~kg}$ ) and average height $182 \mathrm{~cm}$ (SD $7 \mathrm{~cm}$ ). No participant had previously experienced balance problems, central nervous disease or significant injury to the legs, nor were any taking medication. All were asked to refrain from alcohol at least 48 hours prior to testing. Full, informed consent was obtained before any tests were performed, all of which were carried out according to the updated Helsinki Declaration.

\subsection{Equipment}

Anteroposterior and lateral standing postural control were measured with a custom built force platform (dimensions 423mm length x 420mm width x 117mm height above ground), independently recording torques and sheer forces with six degrees of freedom using 6 force transducers with an accuracy of $0.5 \mathrm{~N}$. The data from the force platform were sampled at $50 \mathrm{~Hz}$ and converted into torque and shear force values in three dimensions by a customized computer program which also stored all recordings.

The foam block (dimensions 466mm length x 467mm width $\mathrm{x} 134 \mathrm{~mm}$ height above ground) had a density of $21.3 \mathrm{Kg} / \mathrm{m}^{3}$ and elastic modulus $20900 \mathrm{~N} / \mathrm{m}^{2}$ (Patel et al. 2008a) for detailed description of calculation). Note that the elastic modulus can be considered as an inverse representation of materials compliance. The dimensions of the foam block were slightly larger than the force platform. However, in the measurement setup, the foam was placed on top of the force platform, without contacting the surroundings or ground. A foam block with high elastic modulus was selected in the present study to avoid possible feedback from the firm force platform surface underneath the foam (Patel et al. 2008a).

\subsection{Procedure}

\subsubsection{Posturography procedure}

Participants were asked to stand barefoot on the force platform or on foam placed on top of the force platform, relaxed, with arms folded, in order to maintain consistency and to avoid inappropriate arm and head movements. Participant's heels were $3 \mathrm{~cm}$ apart and feet positioned 
at an angle of $30^{\circ}$ apart open to the front using guidelines on the platform. Participants either focused on a visual target $(6 \times 4 \mathrm{~cm})$ positioned at eye level mounted on the wall at a distance of about $1.5 \mathrm{~m}$, or had their eyes closed when instructed. Participants also listened to music through headphones to reduce external noise sources and sound distractions.

The following tests were performed by all participants in two different test conditions: I) With no hypothermic ischemia of the feet and II) With hypothermic ischemia of the feet.

- $\quad$ Standing on a solid surface with Eyes Open (EO) and Eyes Closed (EC)

- $\quad$ Standing on a compliant foam block with Eyes Open (EO) and Eyes Closed (EC)

In total, 8 tests were performed by each participant.

Each test lasted 120 seconds. The orders of the surface and visual conditions were randomized using a Latin square design to reduce any potential order effect. The hypothermic plantar ischemia tests (cooling) were always performed after the tests without cooling to ensure no contamination. To avoid fatigue and to re-cool the feet between posturography tests involving cooling, there were two minutes rest between each of the 8 tests.

\subsubsection{Cooling Procedure}

Before the cooling tests, participants were seated and rested the entire surface of their feet on ice $\left(-5.3^{\circ} \mathrm{C}\right)$ for 20 minutes. The ice was changed after around 7 and 14 minutes to ensure mechanoreceptive desensitization. Perry et al. (Perry et al. 2000) previously noted that reductions in skin temperature and vibration sensation persisted for more than 20 min after cessation of 15 minutes of active cooling using ice water $\left(0^{\circ} \mathrm{C}\right)$ where the water was not recooled. Following active cooling, all tests were conducted within a 20 minute time-frame. Temperature of the feet was measured under the first metatarsal before cooling, after cooling and after the final balance test with a fast-acting, electronic thermometer. Between tests with 
cooling, participants were asked to rest their feet on ice for 2 minutes in order to maintain desensitization.

\subsubsection{Sensitivity assessment}

Sensitivity tests were performed both prior to posturography testing and after completed posturography testing. The two tests used were vibration perception, which assesses the rapidly adapting mechanoreceptors and tactile sensitivity, which assesses the slowly adapting mechanoreceptors. All sensation scores are based on the mean values from the right foot only due to time constraints with the cooling tests.

Vibration perception of the foot sole was measured using a biothesiometer electronic device (Model EG electronic BioThesiometer, Newbury, Ohio, USA) that generated a $120 \mathrm{~Hz}$ vibration of varying amplitude (in $\mu \mathrm{m}$ ). The vibration was applied to the plantar surface of the first distal phalanx (big toe), the fifth distal phalanx (little toe), the first proximal phalanx (base of big toe), the fifth proximal phalanx (base of little toe) and the tuberosity of calcaneous (heel). Subjects were asked to indicate to the examiner whether they were able to feel the vibration, "Yes" or “No” (Lord et al. 2003; Patel et al. 2009). One reading in ascending intensity and one confirmation reading in descending intensity was made until the subject could no longer feel the vibration. Near the threshold limit, vibration perception was varied by altering the control voltage on the BioThesiometer device in one volt increments or decrements.

Tactile sensitivity was measured with a Semmes-Weinstein pressure aesthesiometer (Semmes-Weinstein Monofilaments, San Jose, USA). The aesthesiometer comprised 20 nylon filaments of equal length, with varying diameter producing a standardized pressure force to the skin (Semmes-Weinstein monofilaments markings 1.65-6.65). The filaments were pressed onto the plantar surface of first distal phalanx (big toe), the fifth distal phalanx (little toe) and the tuberosity of calcaneous (heel). Subjects were instructed that when the filament was placed on 
any of the positions above, they must report to the examiner whether they felt it on the "big toe”, "little toe” or "heel.” Tactile threshold was determined by presenting suprathreshold filaments initially, then applying thinner and thinner filaments until the subject could no longer detect them (Lord et al. 2003; Patel et al. 2009). The examiner then applied thicker filaments until the filament was detected. The filaments were presented in sequence and as the smallest increment or decrement in thickness. The tactile threshold was determined as the average from one ascending and descending step and the calculated tactile threshold is presented using the Semmes-Weinstein monofilament marking definition.

\subsection{Analysis}

Standing balance was assessed by analyzing the variance of the anteroposterior and lateral torque values directly recorded by the force platform. Torque variance values correspond directly to the energy used towards the support surface to maintain stability (Magnusson et al. 1990), where information about energy is required for perceiving the efficiency of standing (Riccio and Stoffregen 1988). For a detailed explanation on torque and its relationship to standing postural control, see Johansson et al. (Johansson et al. 2009).

Anteroposterior and lateral torque from the force platform recordings were divided into three spectral categories; Total torque; torque below $0.1 \mathrm{~Hz}(<0.1 \mathrm{~Hz}$; low-frequency); and torque above $0.1 \mathrm{~Hz}(>0.1 \mathrm{~Hz}$; high-frequency). These separations were used to distinguish between smooth corrective changes of posture (i.e. $<0.1 \mathrm{~Hz}$ ) and fast corrective movements to maintain balance (i.e. >0.1Hz) (Kristinsdottir et al. 2001). Furthermore, the visual and vestibular systems have a cut-off frequency at about $0.1 \mathrm{~Hz}$. The visual system detects movement between around $0.01-0.1 \mathrm{~Hz}$, and supplements the poor sensitivity of the semi-circular canals of the vestibular system within this frequency range (Diener et al. 1986). A fifth-order digital Finite duration Impulse Response (FIR) filter, with filter components selected to avoid aliasing (Proakis and Manolakis 1989), was used for spectral separation of the raw data into low- and high-frequency 
movements, and from this separation low- and high-frequency torque variance values were calculated. Torque variance in all spectral categories was normalized using the subjects' squared height and squared mass before the statistical analysis in order to compensate for the individual differences in height and mass influencing the body sway recorded by a force platform (Fransson et al. 2007; Fransson 2009). The squared nature of the variance algorithm made it necessary to use normalization with squared parameters to achieve unit agreement.

\subsection{Statistical Analysis}

A General Linear Model univariate Analysis of Variance (GLM univariate ANOVA) test was used to determine the effects of standing on foam (Foam) (1 d.f. : standing on foam or on a solid surface); cooling the feet to decrease sensation (Cooling) (1 d.f. : feet cooled or feet with full sensation), and availability of visual information (Vision) (1 d.f. : eyes closed or eyes open) and their interactions on the recorded torque variance values in anteroposterior and lateral directions. The interactions reveal whether the outcome might be influenced by certain combinations of factors. In the analysis, $p$-values $<0.05$ were considered statistically significant.

Wilcoxon, matched-pairs tests were used to investigate in detail the differences in torque variance between test conditions. Non-parametric statistical tests were used as the Shapiro-Wilk test revealed that the values were not normally distributed. Bonferroni correction for multiple comparisons was used and p-values $<0.0167$ were considered statistically significant since 3 pair-wise comparisons were done on each data set (i.e., $\mathrm{p}<0.05 / 3=\mathrm{p}<0.0167$ ).

\section{Results}

\section{1 GLM ANOVA of torque variance}


Table 1 about here

The GLM ANOVA findings presented in table 1 shows that total, low-frequency and highfrequency torque variance in anteroposterior direction were significantly larger when standing on foam compared with standing on solid surface $(\mathrm{p}<0.001)$, see Figures 1 and 2 . Moreover, low-frequency torque variance was significantly larger with decreased sensation by cooling compared with normal sensation $(p=0.019)$. Total $(p=0.011)$ and high-frequency torque variance $(p<0.001)$ were significantly smaller when standing with eyes open compared with eyes closed. None of the interactions reached significance, but there was a clear trend in both total $(p=0.091)$ and high-frequency torque variance $(p<0.066)$ that the stability challenge by standing on foam was handled better with eyes open.

Total, low-frequency and high-frequency torque variance in lateral direction were also significantly larger when standing on foam compared with standing on solid surface $(\mathrm{p}<0.001)$. However, decreased sensation by cooling had no significant influence compared with normal sensation on recorded lateral torque variance. Furthermore, high-frequency torque variance ( $p=0.002)$ was significantly smaller when standing with eyes open compared with eyes closed. None of the interactions reached significance or were close to reaching significant levels.

\subsubsection{Post-Hoc analysis of anteroposterior torque variance}

Figure 1 about here

Figure 1 shows that total and high-frequency torque variance in anteroposterior direction were significantly larger when standing on foam with feet cooled compared with standing on solid surface with and without cooling, both with Eyes Closed and Open. Low-frequency torque 
variance was significantly larger standing on foam with cooling compared with standing on solid surface without cooling with Eyes Closed.

With Eyes Closed, total torque variances were significantly larger when standing on foam without cooling compared with standing on a solid surface with and without cooling. Lowfrequency torque variance was significantly larger standing on foam without cooling compared with standing on solid surface without cooling with Eyes Closed. Additionally, high-frequency torque variance was significantly larger when standing on foam without-cooling compared to standing on solid surface with and without cooling, both with Eyes Closed and Open.

Total and low-frequency torque variance were significantly larger standing on a solid surface with feet cooled compared with standing on solid surface without cooling with Eyes Closed. 
3.2.2 Post-Hoc analysis of lateral torque variance

Figure 2 about here

Figure 2 shows that total, low-frequency and high-frequency torque variances in the lateral direction were significantly larger when standing on foam with and without cooling compared with standing on solid surface with and without cooling, both with Eyes Closed and Eyes Open.

There was no significant difference between standing on a solid surface with and without cooling.

\subsection{Post-Hoc analysis of effects of visual information}

In anteroposterior direction, vision significantly decreased total torque variance when standing on foam with and without cooling and high-frequency torque variance in all tests.

In the lateral direction, vision significantly decreased total torque variance on foam with cooling and high-frequency torque variance when standing on solid surface without cooling and when standing on foam with and without cooling.

3.4 Somatosensory cutaneous mechanoreceptive sensation decrease produced by cooling

Table 2 about here

Mean temperature of the foot sole was $28.4{ }^{\circ} \mathrm{C}$ (Standard Deviation (SD) $2.0^{\circ} \mathrm{C}$ ) before cooling, $15.7^{\circ} \mathrm{C}$ after cooling (SD $2.1^{\circ} \mathrm{C}$ ) and $21.6^{\circ} \mathrm{C}\left(\mathrm{SD} 2.9^{\circ} \mathrm{C}\right)$ after the final posturography test with cooling. When compared to one another, recorded temperature between all conditions yielded a significant difference of $\mathrm{p}<0.001$. 
Vibration perception was significantly decreased by cooling. However, cooling resulted in no significant change in tactile sensitivity (table 5). Mean vibration perception thresholds increased after cooling at the first distal phalanx by $118 \%$, at the little toe by $201 \%$, at the first proximal phalanx by $159 \%$, at the fifth proximal phalanx by $142 \%$ and heel by $98 \%$. Mean tactile sensitivity thresholds increased after cooling at the first distal phalanx by $4 \%$, at the fifth distal phalanx by $10 \%$ and at the heel by $4 \%$.

\section{Discussion}

\subsection{Decreased sensation on a solid surface vs. standing on foam}

It is well-known that when standing on a foam surface, postural control is challenged (Fransson et al. 2007). Foam surfaces are often employed to investigate the relative contributions from the vestibular, visual and somatosensory systems (Enbom et al. 1991; Furman 1995) and are used in the Clinical Test of Sensory Interaction of Balance (CTSIB) i.e. “Foam and Dome” (Nashner et al. 1982; Shumway-Cook and Horak 1986). Foam surfaces have proved to be useful in the clinical evaluation of balance problems, particularly in identifying patients with unilateral and bilateral vestibulopathy (Furman 1995; Fujimoto et al. 2009). The foundation of the CTSIB is largely based upon the principle that standing on a foam surface alters somatosensory information (Nashner et al. 1982; Shumway-Cook and Horak 1986; Horlings et al. 2008; Di Berardino et al. 2009) by decreasing the reliability of plantar cutaneous mechanoreceptive information (Chiang and Wu 1997; Horak and Hlavacka 2001; Jeka et al. 2004; Horlings et al. 2008).

The present study confirmed that standing on foam results in a significant challenge to postural control, increasing body sway significantly in both anteroposterior and lateral directions in the low-frequency $(<0.1 \mathrm{~Hz})$ and high-frequency ranges $(>0.1 \mathrm{~Hz})$. Hypothermic anesthesia mainly increased body sway with Eyes Closed in an anteroposterior direction, but not as strongly as standing on foam, and only in the low-frequency range. The hypothermic 
anesthesia procedures used in this study significantly decreased cutaneous vibration perception by up to $201 \%$, i.e., the rapidly adapting sensation, signaling the fast changes of posture (Kennedy and Inglis 2002), but the hypothermic anesthesia did not significantly change tactile sensitivity, i.e. the slowly adapting sensation signaling the foot sole-surface interaction. Hence, during the hypothermic anesthesia tests in this study, the CNS is likely to still be aware of the pressure distribution under the feet.

The present study revealed large differences in responses between standing on foam and standing with decreased RAMS from cooling. In terms of body sway, hypothermic anesthesia increased total anteroposterior torque variance with Eyes Closed by only $27 \%$ whereas standing on foam increased total anteroposterior torque variance with Eyes Closed by 131\%. Moreover, standing on foam produced a multi-directional postural control challenge (i.e. in both anteroposterior and lateral directions), whereas hypothermic anesthesia predominantly challenged postural control uni-directionally, in anteroposterior direction. In the lateral direction, hypothermic anesthesia even marginally decreased total torque variance by $2 \%$ with Eyes Closed, whereas standing on foam increased total toque variance by $149 \%$ with Eyes Closed. The findings presented also concur with Meyer et al. (Meyer et al. 2004b) who reduced sensation from the entire weight-bearing foot soles of both feet using multiple intradermal injections of anesthesia (2\% lidocaine $\mathrm{HCl}, 1: 8$ sodium bicarbonate, 1:200,000 epinephrine and $12 \mathrm{U} / \mathrm{mg}$ hyaluronidase). Meyer et al. found an increase in Centre of Pressure velocity in an anteroposterior direction with Eyes Closed following anesthesia, but no loss of anteroposterior stability with Eyes Open or lateral stability on a solid surface. However, they found no effect of anaesthesia in Centre of Pressure range.

Spectral separation detailed the differences between standing on foam and standing with decreased RAMS further. In line with Asai et al. (Asai et al. 1990), the present study revealed that hypothermic anesthesia produced an $85 \%$ significantly increased low-frequency torque 
variance in anteroposterior direction on a solid surface, whereas there was a $21 \%$ nonsignificant decrease in high-frequency torque variance with Eyes Closed. Hence, decreased RAMS had effects on postural control suggesting a spectral non-linear relationship between decreased RAMS and corrective postural control responses. A possible explanation for this could be that if a sensory system, which because of the sensory information provided, normally triggers high-frequency corrections, turns quiet or less responsive due to higher reactive thresholds, then this may decrease high-frequency corrections because of reduced information in this spectral range to the CNS to activate a feedback response. Consistent with this hypothesis, there is an increase in activity below $0.1 \mathrm{~Hz}$, suggesting that the forces have to pass higher thresholds to trigger a corrective response. Other possibilities for the decrease in highfrequency torque variance by hypothermic anesthesia could be that the cooling itself restricted body sway or did not produce a sufficient mechanoreceptive decrease to mimic the sensation decrease obtained when standing on foam. Plantar mechanoreceptive information is useful for detecting the limits of stability particularly when balance is perturbed (MacLellan and Patla 2006). Therefore, when the information from the plantar mechanoreceptors is unreliable or difficult to interpret, the CNS may respond by narrowing the margins of stability, forcing less movement by actively using muscular co-contraction during a challenging situation. Moreover, since hypothermic anesthesia does not significantly decrease tactile feedback i.e. foot solesurface information, vertical pressures may be sensed and used as a "map" of pressure distribution and pressure changes under the feet (Kavounoudias et al. 1998). Furthermore, kinesthesia of the ankle joint may substitute for decreased RAMS during unperturbed stance.

In contrast and as confirmed elsewhere (Fransson et al. 2007), standing on foam increases both low-frequency torque variance by about $111 \%$ and high-frequency torque variance by about 155\% with Eyes Closed. Interestingly, recent reports have presented a significant relationship between the body sway in the high-frequency range recorded while standing on 
foam and the mechanical properties of the foam material, i.e., the extent of compression under a given load, and thus, to poorer effectiveness of ankle torque (Patel et al. 2008a; Patel et al. 2008b). Thus, decreased RAMS only marginally affected unperturbed standing balance on solid surface compared with standing on foam.

In this study, the data analyzed were: total recorded stability activity (frequency band $0.0083 \mathrm{~Hz}$ (frequency limit determined by total measurement time) $-25 \mathrm{~Hz}$ (determined by Nyquist criteria for used sample rate) and the recorded data divided into 2 spectral regions, lowfrequency content (between approximately $0.0083 \mathrm{~Hz}$ and $0.1 \mathrm{~Hz}$ ) and high-frequency content (between approximately $0.1 \mathrm{~Hz}$ and $25 \mathrm{~Hz}$ ). Based on the presented findings, it is probable that a more thorough spectral analysis of the recordings may provide more information and identify further differences between standing on foam and standing with decreased RAMS.

\subsection{Effects of decreased sensation when standing on foam}

A possible hypothesis is that hypothermic ischemia produces a different spectral response pattern because the mechanoreceptive sensation decrease is much larger when standing on foam. Thus, an additional sensory decrease by hypothermic anesthesia while standing on foam should in such case produce a response with an additive balance challenge to standing on foam and similar spectral properties. However, when hypothermic anesthesia was combined with standing on foam, findings revealed a small but non-significant additive balance challenge mainly in the lateral direction with Eyes Closed. Moreover, the spectral analysis of body sway revealed that when participants stood on foam with decreased rapidly adapting sensation from hypothermic anesthesia, there were similar but additive spectral properties from both destabilizing methods. The additional effect from the hypothermic anesthesia mainly increased low-frequency torque variance in both anteroposterior and lateral direction and a slight decrease of high-frequency torque variance with Eyes Closed and Open. These findings oppose the hypothesis that hypothermic ischemia produces a different spectral response pattern because 
the mechanoreceptive sensation decrease is much larger when standing on foam. Instead, these findings suggest that the origins of both destabilizing methods are different.

The finding of mainly increased lateral torque variance with decreased sensation on foam is consistent with Meyer et al. (Meyer et al. 2004a) who revealed that during lateral perturbations of balance, decreased plantar sensitivity produced a relative shift in increased compensatory ankle torque. One possible explanation could be that when standing on foam under hypothermic anesthesia, other stabilization strategies may become available due to the compliant nature of foam surfaces. Thus, when standing on a compliant surface, some anteroposterior and lateral movements of the ankle joint will become less restricted and require less effort since foam allows some vertical movements through compression. Interestingly, body leaning on a compliant support surface is by itself sufficient to produce a very different postural control response [(Johansson et al. 2009), figure 8]. Moreover, based on related methodology and considering passive muscle dynamics and neural feedback latencies, Peterka found that the overall behavior in normal subjects was non-linear because gain decreased and phase functions sometimes changed with increasing support surface tilts. Under challenging conditions a more complex positive force feedback control might be needed including proportional, damping and integrative components (Peterka 2002; Peterka 2003). Moreover, the increased available biomechanical motion at the ankle joint in the lateral direction may also give larger gain to any instability caused by cutaneous hypothermic anesthesia in this direction. It also means that when standing on foam, decreased RAMS could be substituted for information from the ankle joint, particularly in the lateral direction, but at a cost of increased biomechanical instability. However, when standing on foam, it is interesting to note that the perturbing effect in the lateral direction is more strongly associated with the mechanical properties of compliant foam surfaces compared with in the anteroposterior direction (Patel et al. 2008a). 
It should be noted that when subjects stand on foam placed on the force platform, the mechanical conditions imposed by the foam material itself, such as its inherent capacity to absorb applied forces and distribute shear forces and torques into different directions or to express these as other postural components such as leaning, may influence the accuracy and properties of values recorded by the force platform below the foam surface. This problem was addressed by assessing the effect of reduced RAMS both when standing on a solid surface and on foam to give indications of possible bias due to altered measurement conditions.

\subsection{Effects of vision}

As found previously, appropriate visual information clearly improved stability, mostly by reducing the requirement of fast corrective movement above $0.1 \mathrm{~Hz}$ (Fransson et al. 2007). Interestingly, during hypothermic anesthesia, which decreased information from RAMS and resulted in increased body sway below $0.1 \mathrm{~Hz}$ predominantly, vision did not significantly reduce body sway in either the anteroposterior or lateral directions. This finding is in line with prior reports showing that vision can only partially compensate for deficits affecting proprioceptive and mechanoreceptive information (Patel et al. 2009).

\section{Conclusions}

Standing on a foam surface is probably the most commonly used method to increase specificity in posturography examinations. However, to draw correct conclusions from the findings using this method, it is important to know how standing on foam causes poorer stability. This study showed that standing on foam is an effective way to challenge postural control and produces a substantial and multi-directional balance perturbation. However, the characteristics of the stability changes caused by decreased cutaneous RAMS through hypothermic anesthesia do not reflect the stability changes when standing on a foam surface. Decreased cutaneous RAMS produced a stability change on solid surface that was different in 
spectral composition, amplitude, direction and that responded differently to vision compared with standing on foam. The findings advocate that standing on foam presents a more complex stability challenge. 


\section{References}

Allum JH, Honegger F (1998) Interactions between vestibular and proprioceptive inputs triggering and modulating human balance-correcting responses differ across muscles. Exp Brain Res 121: 478-494

Asai H, Fujiwara K, Toyama H, Yamashina T, Nara I, Tachino K (1990) The influence of foot soles cooling on standing posture. In: Brandt T, Paulus W, Bles W, Dieterich M, Krafczyk S, Straube A (eds) Disorders of posture and gait. Georg Thieme Verlag Stuttgart, München, pp 198-201

Bloem BR, Allum JH, Carpenter MG, Honegger F (2000) Is lower leg proprioception essential for triggering human automatic postural responses? Exp Brain Res 130: 375-391

Bloem BR, Allum JH, Carpenter MG, Verschuuren JJ, Honegger F (2002) Triggering of balance corrections and compensatory strategies in a patient with total leg proprioceptive loss. Exp Brain Res 142: 91-107

Chiang JH, Wu G (1997) The influence of foam surfaces on biomechanical variables contributing to postural control. Gait Posture 5: 239-245

Di Berardino F, Filipponi E, Barozzi S, Giordano G, Alpini D, Cesarani A (2009) The use of rubber foam pads and "sensory ratios" to reduce variability in static posturography assessment. Gait Posture 29: 158-160

Diener HC, Dichgans J, Guschlbauer B, Bacher M (1986) Role of visual and static vestibular influences on dynamic posture control. Hum neurobiol 5: 105-113

Enbom H, Magnusson M, Pyykkö I (1991) Postural compensation in children with congenital or early acquired bilateral vestibular loss. Ann Otol Rhinol Laryngol 100: 472-478

Fransson PA (2009) Adaptation of Human Postural Control: Learning, sensorimotor and analysis aspects. VDM Verlag, Saarbrücken

Fransson PA, Gomez S, Patel M, Johansson L (2007) Changes in multi-segmented body movements and EMG activity while standing on firm and foam support surfaces. Eur $\mathrm{J}$ Appl Physiol 101: 81-89

Fujimoto C, Murofushi T, Chihara Y, Ushio M, Sugasawa K, Yamaguchi T, Yamasoba T, Iwasaki S (2009) Assessment of diagnostic accuracy of foam posturography for peripheral vestibular disorders: analysis of parameters related to visual and somatosensory dependence. Clin Neurophysiol 120: 1408-1414

Furman JM (1995) Role of posturography in the management of vestibular patients. Otolaryngol Head Neck Surg 112: 8-15

Horak FB, Hlavacka F (2001) Somatosensory loss increases vestibulospinal sensitivity. J Neurophysiol 86: 575-585

Horlings CG, Kung UM, Bloem BR, Honegger F, Van Alfen N, Van Engelen BG, Allum JH (2008) Identifying deficits in balance control following vestibular or proprioceptive loss using posturographic analysis of stance tasks. Clin Neurophysiol 119: 2338-2346

Jeka J, Kiemel T, Creath R, Horak F, Peterka R (2004) Controlling human upright posture: velocity information is more accurate than position or acceleration. J Neurophysiol 92: 2368-2379

Johansson R, Fransson PA, Magnusson M (2009) Optimal coordination and control of posture and movements. J Physiol Paris 103: 159-177

Johansson R, Magnusson M (1991) Human postural dynamics. Crit Rev Biomed Eng 18: 413437

Kavounoudias A, Roll R, Roll JP (1998) The plantar sole is a 'dynamometric map' for human balance control. Neuroreport 9: 3247-3252

Kennedy P, Inglis J (2002) Distribution and behaviour of glabrous cutaneous receptors in the human foot sole. J Physiol 538(3): 995-1002 
Kristinsdottir EK, Fransson PA, Magnusson M (2001) Changes in postural control in healthy elderly subjects are related to vibration sensation, vision and vestibular asymmetry. Acta Otolaryngol 121: 700-706

Kunesch E, Schmidt R, Nordin M, Wallin U, Hagbarth K (1987) Peripheral neural correlates of cutaneous anaesthesia induced by skin cooling in man. Acta Physiol Scand 129(2): 247-257

Lord SR, Menz HB, Tiedemann A (2003) A physiological profile approach to falls risk assessment and prevention. Phys Ther 83: 237-252

MacLellan MJ, Patla AE (2006) Adaptations of walking pattern on a compliant surface to regulate dynamic stability. Exp Brain Res 173: 521-530

Magnusson M, Johansson R, Wiklund J (1990) Galvanically induced body sway in the anteriorposterior plane. Acta Otolaryngol 110: 11-17

Massion J (1994) Postural control system. Curr Opin Neurobiol 4: 877-887

Meyer PF, Oddsson LI, De Luca CJ (2004a) Reduced plantar sensitivity alters postural responses to lateral perturbations of balance. Exp Brain Res 157: 526-536

Meyer PF, Oddsson LI, De Luca CJ (2004b) The role of plantar cutaneous sensation in unperturbed stance. Exp Brain Res 156: 505-512

Nashner LM, Black FO, Wall C (1982) Adaption to altered support and visual conditions during stance: patients with vestibular deficits. J Neurosci 2: 536-544

Patel M, Fransson PA, Lush D, Gomez S (2008a) The effect of foam surface properties on postural stability assessment while standing. Gait Posture 28: 649-656

Patel M, Fransson PA, Lush D, Petersen H, Magnusson M, Johansson R, Gomez S (2008b) The effects of foam surface properties on standing body movement. Acta Otolaryngol: 1-9

Patel M, Gomez S, Berg S, Almbladh P, Lindblad J, Petersen H, Magnusson M, Johansson R, Fransson PA (2008c) Effects of 24-h and 36-h sleep deprivation on human postural control and adaptation. Exp Brain Res 185: 165-173

Patel M, Magnusson M, Kristinsdottir E, Fransson PA (2009) The contribution of mechanoreceptive sensation on stability and adaptation in the young and elderly. Eur $\mathrm{J}$ Appl Physiol 105: 167-173

Perry S, McIllroy W, Maki B (2000) The role of plantar cutaneous mechanoreceptors in the control of compensatory stepping reactions evoked by unpredictable, multi-directional perturbation. Brain Res 877(2): 401-406

Peterka RJ (2002) Sensorimotor integration in human postural control. J Neurophysiol 88: $1097-1118$

Peterka RJ (2003) Simplifying the complexities of maintaining balance. IEEE Eng Med Biol Mag 22: 63-68

Proakis J, Manolakis D (1989) Introduction to Digital Signal Processing. Macmillan, New York

Pyykko I, Jantti P, Aalto H (1990) Postural control in elderly subjects. Age Ageing 19: 215221

Riccio GE, Stoffregen TA (1988) Affordance as contrstraints on the control of stance. Human Movement Science 7: 265-300

Shumway-Cook A, Horak FB (1986) Assessing the influence of sensory interaction of balance. Suggestion from the field. Phys Ther 66: 1548-1550

Stal F, Fransson PA, Magnusson M, Karlberg M (2003) Effects of hypothermic anesthesia of the feet on vibration-induced body sway and adaptation. J Vestib Res 13: 39-52

Wu G, Chiang JH (1997) The significance of somatosensory stimulations to the human foot in the control of postural reflexes. Exp Brain Res 114: 163-169 


\section{Figure Legends}

Figure 1: Anteroposterior torque values with eyes closed (left) and eyes open (right) (mean and (SEM)) for variance of total torque, variance of torque $<0.1 \mathrm{~Hz}$ and variance of torque $>0.1 \mathrm{~Hz}$. The variance values have been normalized with the subject's mass and height. The statistical differences found between the surfaces are marked with asterisks, where $*=$ $\mathrm{p}<0.0167, * *=\mathrm{p}<0.01$ and $* * *=\mathrm{p}<0.001$. After Bonferroni correction for multiple comparisons p-values $<0.0167$ were considered statistically significant.

Figure 2: Lateral torque values with eyes closed (left) and eyes open (right) (mean and (SEM)) for variance of total torque, variance of torque $<0.1 \mathrm{~Hz}$ and variance of torque $>0.1 \mathrm{~Hz}$. The variance values have been normalized with the subject's mass and height. The statistical differences found between the surfaces are marked with asterisks, where $*=\mathrm{p}<0.0167, * *=$ $\mathrm{p}<0.01$ and $* * *=\mathrm{p}<0.001$. 


\section{Tables}

\begin{tabular}{|c|c|c|c|c|c|c|c|}
\hline \multirow{2}{*}{$\begin{array}{l}\text { Anteroposterior } \\
\text { torque variance }\end{array}$} & \multicolumn{7}{|c|}{$p$-value } \\
\hline & Foam & Cooling & Vision & $\begin{array}{l}\text { Foam x } \\
\text { Cooling }\end{array}$ & $\begin{array}{l}\text { Foam x } \\
\text { Vision }\end{array}$ & $\begin{array}{l}\text { Cooling x } \\
\text { Vision }\end{array}$ & $\begin{array}{c}\text { Foam } \mathrm{x} \\
\text { Cooling } \mathrm{x} \\
\text { Vision } \\
\end{array}$ \\
\hline Total & $<0.001[36.5]$ & 0.111 [2.6] & 0.011 [6.6] & 0.235 [1.4] & 0.091 [2.9] & $0.872[0.0]$ & $0.859[0.0]$ \\
\hline$<0.1 \mathrm{~Hz}$ & $<0.001[13.5]$ & $0.019[5.6]$ & $0.873[0.0]$ & 0.101 [2.7] & $0.361[0.8]$ & $0.964[0.0]$ & $0.723[0.1]$ \\
\hline$>0.1 \mathrm{~Hz}$ & $<0.001$ [69.9] & $0.747[0.1]$ & $<0.001[46.9]$ & $0.739[0.1]$ & $0.066[3.5]$ & $0.863[0.0]$ & $0.725[0.1]$ \\
\hline \multirow{2}{*}{$\begin{array}{c}\text { Lateral } \\
\text { torque variance }\end{array}$} & \multicolumn{7}{|c|}{$p$-value } \\
\hline & Foam & Cooling & Vision & $\begin{array}{l}\text { Foam x } \\
\text { Cooling }\end{array}$ & $\begin{array}{l}\text { Foam x } \\
\text { Vision }\end{array}$ & $\begin{array}{l}\text { Cooling x } \\
\text { Vision }\end{array}$ & $\begin{array}{c}\text { Foam } \mathrm{x} \\
\text { Cooling } \mathrm{x} \\
\text { Vision } \\
\end{array}$ \\
\hline Total & $<0.001[75.4]$ & $0.374[0.8]$ & 0.124 [2.4] & $0.810[0.1]$ & 0.319 [1.0] & $0.713[0.1]$ & $0.615[0.3]$ \\
\hline$<0.1 \mathrm{~Hz}$ & $<0.001$ [36.2] & 0.255 [1.3] & $0.773[0.1]$ & $0.914[0.0]$ & $0.813[0.1]$ & $0.291[1.1]$ & $0.921[0.0]$ \\
\hline$>0.1 \mathrm{~Hz}$ & $<0.001[90.1]$ & $0.501[0.5]$ & 0.002 [10.5] & $0.805[0.1]$ & $0.145[2.1]$ & $0.913[0.0]$ & $0.368[0.8]$ \\
\hline
\end{tabular}

Table 1: Statistical evaluation of the anteroposterior and lateral torque variance values using the GLM univariate ANOVA method. "NS" signifies no significant difference. F values are presented in the squared parenthesis. 


\section{Mechanoreceptive sensation}

\begin{tabular}{ccccc}
\cline { 3 - 5 } & & $\begin{array}{c}\text { Control } \\
\text { (SD) }\end{array}$ & $\begin{array}{c}\text { Cooling } \\
\text { (SD) }\end{array}$ & P-Val \\
\hline & Big toe & $0.64(0.28)$ & $1.39(1.15)$ & 0.001 \\
Vibration & Little toe & $0.55(0.37)$ & $1.66(1.48)$ & $<0.001$ \\
$\begin{array}{c}\text { Perception } \\
{[\boldsymbol{\mu m}]}\end{array}$ & Base of big toe & $0.33(0.21)$ & $0.85(0.68)$ & 0.001 \\
& Base of little toe & $0.33(0.23)$ & $0.80(0.67)$ & 0.001 \\
& Heel & $0.28(0.17)$ & $0.56(0.44)$ & 0.010 \\
\hline $\begin{array}{c}\text { Tactile } \\
\text { Sensitivity } \\
{[S-W *]}\end{array}$ & Big toe & $4.02(0.52)$ & $4.16(0.84)$ & 0.354 \\
& Little toe & $3.49(0.54)$ & $3.85(0.51)$ & 0.117 \\
\hline
\end{tabular}

Table 2: Vibration perception and tactile sensitivity (mean and standard deviation (SD)) measurements before and after cooling. P-values show the significant differences between before and after cooling are presented. "NS" signifies no significant difference. *Semmes-Weinstein monofilament marking. 